\title{
STABLE AND TOTAL FENCHEL DUALITY FOR CONVEX OPTIMIZATION PROBLEMS IN LOCALLY CONVEX SPACES*
}

\author{
CHONG LI $^{\dagger}$, DONGHUI FANG ${ }^{\ddagger}$, GENARO LÓPEZ§, AND MARCO A. LÓPEZ
}

\begin{abstract}
We consider the optimization problem $\left(P_{A}\right) \inf _{x \in X}\{f(x)+g(A x)\}$ where $f$ and $g$ are proper convex functions defined on locally convex Hausdorff topological vector spaces $X$ and $Y$, respectively, and $A$ is a linear operator from $X$ to $Y$. By using the properties of the epigraph of the conjugated functions, some sufficient and necessary conditions for the strong Fenchel duality and the strong converse Fenchel duality of $\left(P_{A}\right)$ are provided. Sufficient and necessary conditions for the stable Fenchel duality and for the total Fenchel duality are also derived.
\end{abstract}

Key words. stable and total Fenchel duality, converse duality, convex optimization in locally convex spaces

AMS subject classifications. Primary, 49N15, 90C25; Secondary, 90C46

DOI. $10.1137 / 080734352$

1. Introduction. Let $X$ and $Y$ be real locally convex Hausdorff topological vector spaces, whose respective dual spaces, $X^{*}$ and $Y^{*}$, are endowed with the weak*topologies $w^{*}\left(X^{*}, X\right)$ and $w^{*}\left(Y^{*}, Y\right)$. Let $f: X \rightarrow \mathbb{R} \cup\{+\infty\}, g: Y \rightarrow \mathbb{R} \cup\{+\infty\}$ be proper convex functions, and let $A: X \rightarrow Y$ be a linear operator such that $A(\operatorname{dom} f) \cap \operatorname{dom} g \neq \emptyset$. We consider the primal problem

$$
\left(P_{A}\right) \inf _{x \in X}\{f(x)+g(A x)\}
$$

and its associated dual problem

$$
\left(D_{A}\right) \sup _{y^{*} \in Y_{A}^{*}}\left\{-f^{*}\left(-A^{*} y^{*}\right)-g^{*}\left(y^{*}\right)\right\},
$$

where $f^{*}$ and $g^{*}$ are the Fenchel conjugates of $f$ and $g$, respectively, and $A^{*}: Y_{A}^{*} \rightarrow X^{*}$ stands for the adjoint operator, where $Y_{A}^{*}$ is the subspace of $Y^{*}$ such that $y^{*} \in Y_{A}^{*}$ if and only if $A^{*} y^{*}$ defined by $\left\langle A^{*} y^{*}, \cdot\right\rangle=\left\langle y^{*}, A(\cdot)\right\rangle$ is continuous on $X$. Note that, in general, $Y_{A}^{*}$ is not the whole space $Y^{*}$ because $A$ is not necessarily continuous. From now on, we shall identify the dual pair $\left(P_{A}\right)$ and $\left(D_{A}\right)$ with the triple $(f, g, A)$.

It is well known that the optimal values of these problems, $v\left(P_{A}\right)$ and $v\left(D_{A}\right)$, respectively, satisfy the so-called weak duality, i.e., $v\left(P_{A}\right) \geq v\left(D_{A}\right)$, but a duality gap

\footnotetext{
* Received by the editors September 4, 2008; accepted for publication (in revised form) April 17, 2009; published electronically July 30, 2009.

http://www.siam.org/journals/siopt/20-2/73435.html

${ }^{\dagger}$ Department of Mathematics, Zhejiang University, Hangzhou 310027, People's Republic of China (cli@zju.edu.cn). This author was supported in part by the National Natural Science Foundation of China (grants 10671175, 10731060) and the DGES, grant SAB 2006-0195, Spain.

‡Department of Mathematics, Zhejiang University, Hangzhou 310027, People’s Republic of China; College of Mathematics and Computer Science, Jishou University, Jishou 416000, People's Republic of China (dh_fang@jsu.edu.cn). This author was supported in part by Grant MTM-2006-13997-C0201 .

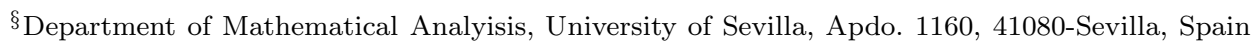
(glopez@us.es). This author was supported in part by Grant MTM-2006-13997-C02-01.

ฯDepartment of Statistics and Operations Research, University of Alicante, 03071 Alicante, Spain (marco.antonio@ua.es). This author was supported in part by Grant MTM2008-06695-C03-01/MTM (Ministerio de Ciencia e Innovación, Spain).
} 
may occur; i.e., we can have $v\left(P_{A}\right)>v\left(D_{A}\right)$. A challenge in convex analysis has been to give sufficient conditions which guarantee strong Fenchel duality, i.e., the situation when there is no duality gap and the dual problem has at least an optimal solution. Several interiority-type conditions were given in order to preclude the existence of such a duality gap in different settings (see, for instance, $[8,15,19]$, and [21, Theorem 2.8.3]). Recently, Bots et al. introduced in [2] a new weaker interiority condition, and established the strong Fenchel duality under the new condition (see (4.21) in section 4).

Taking inspiration from Burachik and Jeyakumar $[9,10]$, some authors approached the strong duality problem by using some properties of the epigraphs of the functions $f^{*}$ and $g^{*}$ (see, for instance, [7, 11]). In particular, when $f$ and $g$ are proper lower semicontinuous (lsc, in brief) convex functions and $A$ is a linear continuous operator, Bot and Wanka [7] gave a sufficient condition of this type, guaranteeing strong duality between $\left(P_{A}\right)$ and $\left(D_{A}\right)$.

Another related interesting problem is the converse strong Fenchel duality, which corresponds to the situation in which $v\left(P_{A}\right)=v\left(D_{A}\right)$ and $\left(P_{A}\right)$ has at least an optimal solution. This problem was considered in [7] for the case in which $f$ and $g$ are proper, convex, lsc functions and $A$ is a continuous linear operator, in which case the converse strong duality problem is equivalently represented as a strong duality problem for some closely related optimization problems. This is no longer true for the general case, i.e., when $f$ and $g$ are not necessarily lsc and $A$ is not necessarily continuous. This makes the converse duality problem more complicated and interesting, and this is why we deal with this problem here.

In this paper we study different kinds of duality between $\left(P_{A}\right)$ and $\left(D_{A}\right)$ in the most general setting, namely when $f$ and $g$ are proper convex functions (not necessarily lsc) and $A$ is a linear operator (not necessarily continuous). We introduce some new regularity conditions such as $(C C)_{A}$ and $(F R C)_{A}$, and establish the relationships between them and the condition $(R C)_{A}$ defined in [7]. In terms of these new regularity conditions, we provide sufficient and necessary conditions for the strong Fenchel duality, the stable Fenchel duality, and the total Fenchel duality between $\left(P_{A}\right)$ and $\left(D_{A}\right)$, which generalize the results in [7] and [2]. Similar characterization results for the converse strong Fenchel duality, the converse stable Fenchel duality, and the converse total Fenchel duality are also given in the paper, improving and extending the corresponding results in [7].

2. Notations and preliminary results. The notation used in the present paper is standard (cf. $[12,14,21]$ ). In particular, we assume throughout the whole paper that $X$ and $Y$ are real locally convex Hausdorff topological vector spaces, and let $X^{*}$ denote the dual space, endowed with the weak ${ }^{*}$-topology $w^{*}\left(X^{*}, X\right)$. By $\left\langle x^{*}, x\right\rangle$ we shall denote the value of the functional $x^{*} \in X^{*}$ at $x \in X$, i.e., $\left\langle x^{*}, x\right\rangle=x^{*}(x)$. The zero of each of the involved spaces will be indistinctly represented by 0 .

If $Z \subset X$, the interior, closure, convex hull, and the convex conical hull of $Z$ are denoted by $\operatorname{int} Z, \operatorname{cl} Z, \operatorname{co} Z$, and cone $Z$, respectively. The indicator function $\delta_{Z}: X \rightarrow \overline{\mathbb{R}}:=\mathbb{R} \cup\{+\infty\}$ and the support function $\sigma_{Z}: X^{*} \rightarrow \overline{\mathbb{R}}$ of the nonempty set $Z$ are the proper convex functions, respectively, defined by

$$
\delta_{Z}(x):=\left\{\begin{array}{cc}
0, & x \in Z \\
+\infty, & \text { otherwise }
\end{array}\right.
$$

and

$$
\sigma_{Z}\left(x^{*}\right):=\sup _{x \in Z}\left\langle x^{*}, x\right\rangle
$$


Let $f: X \rightarrow \overline{\mathbb{R}}$ be a proper convex function. The effective domain and the epigraph of $f$ are the nonempty sets defined by

$$
\operatorname{dom} f:=\{x \in X: f(x)<+\infty\}
$$

and

$$
\text { epi } f:=\{(x, r) \in X \times \mathbb{R}: f(x) \leq r\} .
$$

The closure of $f$ is the convex function denoted by $\mathrm{cl} f$, and whose epigraph is

$$
\operatorname{epi}(\operatorname{cl} f)=\operatorname{cl}(\text { epi } f)
$$

where the topological closure is taken with respect to the product topology. The conjugate function of $f$ is the function $f^{*}: X^{*} \rightarrow \overline{\mathbb{R}}$ defined by

$$
f^{*}\left(x^{*}\right):=\sup \left\{\left\langle x^{*}, x\right\rangle-f(x): x \in X\right\} .
$$

By [21, Theorem 2.3.4], if $\mathrm{cl} f$ is proper, then the following equality holds:

$$
f^{* *}=\operatorname{cl} f .
$$

Let $x \in \operatorname{dom} f$. The subdifferential of $f$ at $x$ is the convex set defined by

$$
\partial f(x):=\left\{x^{*} \in X^{*}: f(x)+\left\langle x^{*}, y-x\right\rangle \leq f(y) \text { for each } y \in X\right\} .
$$

Moreover, the Young equality holds (cf. [21, Theorem 2.4.2(iii)]):

$$
f(x)+f^{*}\left(x^{*}\right)=\left\langle x^{*}, x\right\rangle \Longleftrightarrow x^{*} \in \partial f(x) .
$$

As a consequence of that,

$$
\left(x^{*},\left\langle x^{*}, x\right\rangle-f(x)\right) \in \operatorname{epi} f^{*} \quad \text { for all } x^{*} \in \partial f(x) .
$$

Given two proper functions $g, h: X \rightarrow \overline{\mathbb{R}}$, we define the infimal convolution of $g$ and $h$ as the function $g \square h: X \rightarrow \mathbb{R} \cup\{ \pm \infty\}$ given by

$$
(g \square h)(x):=\inf _{z \in X}\{g(z)+h(x-z)\} .
$$

$g \square h$ is said to be exact at some $x \in X$ if there is $z \in X$ such that $(g \square h)(x)=$ $g(z)+h(x-z)$. Note that if $g \square h$ is exact at $x$, then $(g \square h)(x)>-\infty$; while if $(g \square h)(x)=+\infty$, then $g \square h$ is exact at $x$.

The following notion of Cartesian product map is used in [7]:

Definition 2.1. Let $M_{1}, M_{2}, N_{1}, N_{2}$ be nonempty sets and consider maps $F$ : $M_{1} \rightarrow M_{2}$ and $G: N_{1} \rightarrow N_{2}$. We denote by $F \times G: M_{1} \times N_{1} \rightarrow M_{2} \times N_{2}$ the map defined by

$$
(F \times G)(x, y):=(F(x), G(y)) .
$$

DeFinition 2.2. Let $A: X \rightarrow Y$ be a linear operator, and consider the function $h: X \rightarrow \overline{\mathbb{R}}$. Then, the function $A h: Y \rightarrow \mathbb{R} \cup\{ \pm \infty\}$ defined by

$$
(A h)(y):=\inf \{h(x): A x=y\}
$$

Copyright $@$ by SIAM. Unauthorized reproduction of this article is prohibited. 
is called the image of $h$ under $A$. Here we adopt the convention that $(A h)(y)=+\infty$ if $A^{-1}(y):=\{x \in X: A x=y\}$ is empty.

For the whole paper, we endow $X^{*} \times \mathbb{R}$ with the product topology of $w^{*}\left(X^{*}, X\right)$ and the usual Euclidean topology. The following lemma characterizes the epigraph of the conjugate of the sum of two functions. Part (i) is a consequence of the RockafellarMoreau theorem (cf. $[18,20]$ ), and as in [7, Theorem 2.4].

Lemma 2.3. Let $g, h: X \rightarrow \overline{\mathbb{R}}$ be proper convex functions such that $\operatorname{dom} g \cap$ $\operatorname{dom} h \neq \emptyset$.

(i) If $g, h: X \rightarrow \overline{\mathbb{R}}$ are lower semicontinuous, then

$$
\operatorname{epi}(g+h)^{*}=\operatorname{cl}\left(\operatorname{epi}\left(g^{*} \square h^{*}\right)\right)=\operatorname{cl}\left(\operatorname{epi} g^{*}+\operatorname{epi} h^{*}\right) .
$$

(ii) If either $g$ or $h$ is continuous at some $x_{0} \in \operatorname{dom} g \cap \operatorname{dom} h$, then

$$
\text { epi }(g+h)^{*}=\operatorname{epi} g^{*}+\text { epi } h^{*} \text {. }
$$

Proof. Part (i) is already stated and, so we shall prove part (ii). In virtue of [21, Theorem 2.8.7], the given assumption implies that $(g+h)^{*}=g^{*} \square h^{*}$ and $g^{*} \square h^{*}$ is exact at every $p \in X^{*}$. Then the result is clear from [7, Proposition 2.2].

3. New regularity conditions. Let $f: X \rightarrow \overline{\mathbb{R}}, g: Y \rightarrow \overline{\mathbb{R}}$ be proper convex functions and $A: X \rightarrow Y$ a linear operator such that $A(\operatorname{dom} f) \cap \operatorname{dom} g \neq \emptyset$. Next we introduce the regularity condition $(C C)_{A}$ for the triple $(f, g, A)$, which is crucial in our approach. To this aim, we shall consider the identity map $\operatorname{id}_{\mathbb{R}}$ on $\mathbb{R}$, and the image set $\left(A^{*} \times \operatorname{id}_{\mathbb{R}}\right)\left(\right.$ epi $\left.g^{*}\right)$ of epi $g^{*}$ through the map $A^{*} \times \operatorname{id}_{\mathbb{R}}: Y_{A}^{*} \times \mathbb{R} \rightarrow X^{*} \times \mathbb{R}$, that is,

$$
\left(x^{*}, r\right) \in\left(A^{*} \times \operatorname{id}_{\mathbb{R}}\right)\left(\text { epi } g^{*}\right) \Leftrightarrow\left\{\begin{array}{l}
\exists y^{*} \in Y_{A}^{*} \text { such that }\left(y^{*}, r\right) \in \text { epi } g^{*} \\
\text { and } A^{*} y^{*}=x^{*}
\end{array}\right.
$$

In this section, we always assume that $\mathrm{cl} f, \operatorname{cl} g$, and $\operatorname{cl}((\operatorname{cl} g) \circ A)$ are proper functions.

Definition 3.1. The triple $(f, g, A)$ is said to satisfy the condition $(C C)_{A}$ if

$$
\text { epi }(f+g \circ A)^{*} \subseteq \text { epi } f^{*}+\left(A^{*} \times \operatorname{id}_{\mathbb{R}}\right)\left(\text { epi } g^{*}\right) \text {. }
$$

Lemma 3.2. The following inclusion relation holds automatically:

$$
\text { epi } f^{*}+\left(A^{*} \times \operatorname{id}_{\mathbb{R}}\right)\left(\operatorname{epi} g^{*}\right) \subseteq \operatorname{epi}(f+g \circ A)^{*} .
$$

Furthermore, if $A$ is continuous, then the following assertion holds:

$$
\operatorname{cl}\left(\left(A^{*} \times \operatorname{id}_{\mathbb{R}}\right)\left(\operatorname{epi} g^{*}\right)\right)=\operatorname{epi}((\operatorname{cl} g) \circ A)^{*} \subseteq \operatorname{epi}(g \circ A)^{*} .
$$

Proof. Let $\left(x_{1}^{*}, r_{1}\right) \in$ epi $f^{*}$ and $\left(x_{2}^{*}, r_{2}\right) \in\left(A^{*} \times \operatorname{id}_{\mathbb{R}}\right)\left(\right.$ epi $\left.g^{*}\right)$. Then there exists $y^{*} \in Y_{A}^{*}$ such that $A^{*} y^{*}=x_{2}^{*}$ and $g^{*}\left(y^{*}\right) \leq r_{2}$. Hence,

$$
\begin{aligned}
(f+g \circ A)^{*}\left(x_{1}^{*}+x_{2}^{*}\right) & =\sup _{x \in X}\left\{\left\langle x_{1}^{*}+x_{2}^{*}, x\right\rangle-(f+g \circ A)(x)\right\} \\
& \leq \sup _{x \in X}\left\{\left\langle x_{1}^{*}, x\right\rangle-f(x)\right\}+\sup _{x \in X}\left\{\left\langle x_{2}^{*}, x\right\rangle-(g \circ A)(x)\right\} \\
& =\sup _{x \in X}\left\{\left\langle x_{1}^{*}, x\right\rangle-f(x)\right\}+\sup _{x \in X}\left\{\left\langle y^{*}, A x\right\rangle-(g \circ A)(x)\right\} \\
& \leq f^{*}\left(x_{1}^{*}\right)+g^{*}\left(y^{*}\right) \leq r_{1}+r_{2} .
\end{aligned}
$$

This implies that $\left(x_{1}^{*}+x_{2}^{*}, r_{1}+r_{2}\right) \in \operatorname{epi}(f+g \circ A)^{*}$ and so (3.2) holds. 
Suppose that $A$ is continuous. By [3, Lemma 1] (see also (4) in [7]), one has that

$$
\operatorname{cl}\left(\left(A^{*} \times \operatorname{id}_{\mathbb{R}}\right)\left(\operatorname{epi}(\operatorname{cl} g)^{*}\right)\right)=\operatorname{epi}((\operatorname{cl} g) \circ A)^{*} .
$$

Now the equality in (3.3) is clear since $(\mathrm{cl} g)^{*}=g^{*}$. The inclusion in (3.3) is trivial because $(\mathrm{cl} g) \circ A \leq g \circ A$. The proof is complete.

Thus the following result is straightforward.

LEMma 3.3. The condition $(C C)_{A}$ holds if and only if

$$
\text { epi }(f+g \circ A)^{*}=\operatorname{epi} f^{*}+\left(A^{*} \times \operatorname{id}_{\mathbb{R}}\right)\left(\text { epi } g^{*}\right) \text {. }
$$

When $X=Y$ and $A=\operatorname{id}_{X}$, we get $A^{*}=\operatorname{id}_{X^{*}}$ (as a consequence of that $X$ separates points of $\left.\left(X^{*}, w^{*}\left(X^{*}, X\right)\right)\right)$, and (3.4) reads

$$
\text { epi }(f+g)^{*}=\text { epi } f^{*}+\text { epi } g^{*} \text {. }
$$

Definition 3.4. The triple $(f, g, A)$ satisfies the condition $(C l S)_{A}$ if

$$
\operatorname{cl}(f+g \circ A) \leq(\operatorname{cl} f)+\operatorname{cl}((\operatorname{cl} g) \circ A) .
$$

Then we have the following lemma.

Lemma 3.5. $(C l S)_{A}$ is equivalent to the condition

$$
\operatorname{cl}(f+g \circ A)=(\operatorname{cl} f)+\operatorname{cl}((\operatorname{cl} g) \circ A) .
$$

Proof. From $\operatorname{cl} f \leq f$ and $\operatorname{cl}((\operatorname{cl} g) \circ A) \leq g \circ A$, we deduce that

$$
(\operatorname{cl} f)+\operatorname{cl}((\operatorname{cl} g) \circ A) \leq f+g \circ A,
$$

and the lower semicontinuity of $(\operatorname{cl} f)+\operatorname{cl}((\operatorname{cl} g) \circ A)$ entails

$$
(\operatorname{cl} f)+\operatorname{cl}((\operatorname{cl} g) \circ A) \leq \operatorname{cl}(f+g \circ A) .
$$

The proof is complete.

If $A$ is continuous, (3.7) is equivalent to the condition used in [13, Theorem 13]. Using [13, Lemma 15], we have the following proposition, which presents a sufficient condition ensuring $(C C)_{A}$ for the triple $(f, g, A)$ when $A$ is continuous.

Proposition 3.6. Suppose that $A$ is continuous and $g$ is continuous at $A x_{0}$ for some point $x_{0} \in A^{-1}(\operatorname{dom} g) \cap \operatorname{dom} f$. Then the triple $(f, g, A)$ satisfies the condition $(C C)_{A}$.

Proof. By [13, Lemma 15], we have that

$$
\operatorname{cl}(f+g \circ A)=(\operatorname{cl} f)+((\operatorname{cl} g) \circ A) .
$$

Furthermore, by [21, Theorem 2.8.3(iii)], one has that for all $x^{*} \in X^{*}$,

$$
((\operatorname{cl} f)+(\operatorname{cl} g) \circ A)^{*}\left(x^{*}\right)=\min \left\{(\operatorname{cl} f)^{*}\left(x^{*}-A^{*} y^{*}\right)+(\operatorname{cl} g)^{*}\left(y^{*}\right): y^{*} \in Y^{*}\right\},
$$

which is equivalent to

$$
\operatorname{epi}((\operatorname{cl} f)+(\operatorname{cl} g) \circ A)^{*}=\operatorname{epi} f^{*}+\left(A^{*} \times \operatorname{id}_{\mathbb{R}}\right)\left(\operatorname{epi}(\operatorname{cl} g)^{*}\right)
$$

thanks to [7, Theorem 3.1 (i)]. This together with (3.8) implies that

$$
\text { epi }(f+g \circ A)^{*}=\text { epi } f^{*}+\left(A^{*} \times \operatorname{id}_{\mathbb{R}}\right)\left(\text { epi } g^{*}\right) .
$$

Hence, the proof is complete. 
The following regularity condition is key in [7]:

DEFINITION 3.7. Under the assumptions that $f$ and $g$ are proper, convex, and lsc functions, and that $A$ is a continuous linear operator, the triple $(f, g, A)$ satisfies the condition $(R C)_{A}$ if

$$
\text { epi } f^{*}+\left(A^{*} \times \operatorname{id}_{\mathbb{R}}\right)\left(\text { epi } g^{*}\right) \text { is closed. }
$$

The following proposition describes the relationship among conditions $(C C)_{A}$, $(R C)_{A}$, and $(C l S)_{A}$. For simplicity, in the special case when $X=Y$ and $A=\operatorname{id}_{X}$ (the identity on $X$ ), we write $(C C),(R C)$, and $(C l S)$, respectively, for $(C C)_{A},(R C)_{A}$, and $(C l S)_{A}$.

Proposition 3.8. Suppose that $A$ is continuous. Then the following equivalence holds:

$$
(C C)_{A} \Longleftrightarrow\left[(R C)_{A} \quad \&(C l S)_{A}\right]
$$

Proof. We shall prove that

$$
(C l S)_{A} \Leftrightarrow \operatorname{epi}(f+g \circ A)^{*}=\operatorname{cl}\left(\operatorname{epi} f^{*}+\left(A^{*} \times \operatorname{id}_{\mathbb{R}}\right)\left(\text { epi } g^{*}\right)\right) .
$$

Once we have established the equivalence (3.11), (3.10) is a consequence of Lemma 3.3. The following equivalence is a consequence of Lemma 3.5:

$$
\begin{aligned}
(C l S)_{A} & \Leftrightarrow(f+g \circ A)^{*}=((\operatorname{cl} f)+\operatorname{cl}((\operatorname{cl} g) \circ A))^{*} \\
& \Leftrightarrow \operatorname{epi}(f+g \circ A)^{*}=\operatorname{epi}((\operatorname{cl} f)+\operatorname{cl}((\operatorname{cl} g) \circ A))^{*} .
\end{aligned}
$$

In order to prove (3.11), it suffices to check that

$$
\operatorname{epi}((\operatorname{cl} f)+\operatorname{cl}((\operatorname{cl} g) \circ A))^{*}=\operatorname{cl}\left(\operatorname{epi} f^{*}+\left(A^{*} \times \operatorname{id}_{\mathbb{R}}\right)\left(\operatorname{epi} g^{*}\right)\right) .
$$

By the assumption $A(\operatorname{dom} f) \cap \operatorname{dom} g \neq \emptyset$ made at the beginning of this section, one can easily see that $\operatorname{dom}(\operatorname{cl} f) \cap \operatorname{dom}(\operatorname{cl}((\operatorname{cl} g) \circ A)) \neq \emptyset$. Thus Lemma 2.3 is applicable and

$$
\begin{aligned}
\operatorname{epi}((\operatorname{cl} f)+\operatorname{cl}((\operatorname{cl} g) \circ A))^{*} & =\operatorname{cl}\left(\operatorname{epi}(\operatorname{cl} f)^{*}+\operatorname{epi}(\operatorname{cl}((\operatorname{cl} g) \circ A))^{*}\right) \\
& =\operatorname{cl}\left(\operatorname{epi} f^{*}+\operatorname{epi}((\operatorname{cl} g) \circ A)^{*}\right) .
\end{aligned}
$$

Combining this with (3.3), we get certainly (3.13):

$$
\begin{aligned}
\operatorname{epi}((\operatorname{cl} f)+\operatorname{cl}((\operatorname{cl} g) \circ A))^{*} & =\operatorname{cl}\left(\operatorname{epi} f^{*}+\operatorname{epi}((\operatorname{cl} g) \circ A)^{*}\right) \\
& =\operatorname{cl}\left(\operatorname{epi} f^{*}+\operatorname{cl}\left(\left(A^{*} \times \operatorname{id}_{\mathbb{R}}\right)\left(\text { epi } g^{*}\right)\right)\right) \\
& =\operatorname{cl}\left(\operatorname{epi} f^{*}+\left(A^{*} \times \operatorname{id}_{\mathbb{R}}\right)\left(\text { epi } g^{*}\right)\right) .
\end{aligned}
$$

Note that when $f$ and $g$ are lsc functions and $A$ is continuous, $(C l S)_{A}$ holds automatically. Therefore, the following corollary is obvious.

Corollary 3.9. Suppose that $f$ and $g$ are proper, convex, and lsc functions and that $A$ is a continuous linear operator. Then the following equivalence holds:

$$
(C C)_{A} \Longleftrightarrow(R C)_{A} .
$$

The following proposition can be found in [6], where the same sum rule of $\epsilon$ subdifferential was obtained for general functions (not necessarily convex). Here, as an application of Proposition 3.8, we give a direct proof of this proposition.

Copyright (c) by SIAM. Unauthorized reproduction of this article is prohibited. 
Proposition 3.10. Suppose that the functions $f$ and $g$ satisfy the condition (3.5). Then, for each $x \in \operatorname{dom} g \cap \operatorname{dom} f$, one has

$$
\partial(f+g)(x)=\partial f(x)+\partial g(x) .
$$

Proof. Let $x \in \operatorname{dom} g \cap \operatorname{dom} f$. Since we always have $\partial f(x)+\partial g(x) \subseteq \partial(f+g)(x)$, we shall assume that $\partial(f+g)(x) \neq \emptyset$. By [21, Theorem 2.4.1],

$$
(f+g)(x)=(\operatorname{cl}(f+g))(x) \quad \text { and } \quad \partial(f+g)(x)=\partial(\operatorname{cl}(f+g))(x) .
$$

By Proposition 3.8, $(C l S)$ and $(R C)$ hold (they are conjointly equivalent to $(C C)$, i.e., to $(3.5))$. Hence, $(C l S)$ gives rise to

$$
\operatorname{cl}(f+g)=\operatorname{cl} f+\operatorname{cl} g .
$$

Since $(f+g)(x)=(\operatorname{cl}(f+g))(x)$ by $(3.16)$, and since $(\operatorname{cl} f)(x) \leq f(x)$ and $(\operatorname{cl} g)(x) \leq$ $g(x)$, it follows that

$$
(\operatorname{cl} f)(x)=f(x) \quad \text { and } \quad(\operatorname{cl} g)(x)=g(x) .
$$

Consequently,

$$
\partial f(x)=\partial(\operatorname{cl} f)(x) \quad \text { and } \quad \partial g(x)=\partial(\operatorname{cl} g)(x) .
$$

Using (3.16), (3.17), and applying [7, Theorem 3.1(ii)], we conclude that

$$
\begin{aligned}
\partial(f+g)(x) & =\partial(\operatorname{cl} f+\operatorname{cl} g)(x) \\
& =\partial(\operatorname{cl} f)(x)+\partial(\operatorname{cl} g)(x) \\
& =\partial f(x)+\partial g(x) . \quad
\end{aligned}
$$

For the general case, the arguments for the proof of [7, Theorem 3.1(ii)] can also be used to prove the corresponding version of the Moreau-Rockafellar formula for the subdifferential of the sum. Recall that, as usual, for a subset $D \subseteq Y^{*}, A^{*} D$ reads as

$$
A^{*} D=\left\{A^{*} y^{*}: y^{*} \in Y_{A}^{*} \cap D\right\},
$$

with the convention that $A^{*} D=\emptyset$ if $Y_{A}^{*} \cap D=\emptyset$.

Proposition 3.11. Suppose that $(f, g, A)$ satisfies $(C C)_{A}$. Then, for each $x \in$ $A^{-1}(\operatorname{dom} g) \cap \operatorname{dom} f$, one gets

$$
\partial(f+g \circ A)(x)=\partial f(x)+A^{*} \partial g(A x) .
$$

4. Stable Fenchel duality. This section is devoted to the study of the strong duality between the primal problem and its Fenchel dual; namely, the property that both optimal values coincide and the dual problem has at least an optimal solution. Recall that $X$ and $Y$ are real locally convex Hausdorff topological vector spaces, $A: X \rightarrow Y$ is a linear operator, and that $f: X \rightarrow \overline{\mathbb{R}}$ and $g: Y \rightarrow \overline{\mathbb{R}}$ are proper convex functions such that $A(\operatorname{dom} f) \cap \operatorname{dom} g \neq \emptyset$.

Given $p \in X^{*}$, we consider the following convex optimization problem with a linear perturbation

$$
\left(P_{(A, p)}\right): \quad \inf _{x \in X}\{f(x)+g(A x)-\langle p, x\rangle\},
$$

Copyright (c) by SIAM. Unauthorized reproduction of this article is prohibited. 
and the corresponding dual problem

$$
\left(D_{(A, p)}\right): \quad \sup _{y^{*} \in Y_{A}^{*}}\left\{-f^{*}\left(p-A^{*} y^{*}\right)-g^{*}\left(y^{*}\right)\right\} .
$$

Let us denote by $v\left(P_{(A, p)}\right)$ and $v\left(D_{(A, p)}\right)$ the optimal values of problem $\left(P_{(A, p)}\right)$ and $\left(D_{(A, p)}\right)$, respectively, that is

$$
v\left(P_{(A, p)}\right)=\inf _{x \in X}\{f(x)+g(A x)-\langle p, x\rangle\},
$$

and

$$
v\left(D_{(A, p)}\right)=\sup _{y^{*} \in Y_{A}^{*}}\left\{-f^{*}\left(p-A^{*} y^{*}\right)-g^{*}\left(y^{*}\right)\right\} .
$$

In particular, when $p=0$, problems $\left(P_{(A, p)}\right)$ and $\left(D_{(A, p)}\right)$ are just the problems $\left(P_{A}\right)$ and $\left(D_{A}\right)$. It is easy to see that the weak dual inequality holds:

$$
v\left(D_{(A, p)}\right) \leq v\left(P_{(A, p)}\right) \text { for all } p \in X^{*} .
$$

We have the following expressions:

$$
v\left(P_{A}\right)=-(f+g \circ A)^{*}(0),
$$

and

$$
v\left(D_{A}\right)=-\left(f^{*} \square A^{*} g^{*}\right)(0) .
$$

Consequently, the weak dual inequality is equivalent to

$$
(f+g \circ A)^{*}(0) \leq\left(f^{*} \square A^{*} g^{*}\right)(0),
$$

and also to

$$
\text { epi }\left(f^{*} \square A^{*} g^{*}\right) \cap(\{0\} \times \mathbb{R}) \subseteq \text { epi }(f+g \circ A)^{*} \cap(\{0\} \times \mathbb{R}) .
$$

In fact, (4.6) is direct, while (4.7) holds because

$$
\begin{aligned}
\left(f^{*} \square A^{*} g^{*}\right)(0) & =\inf _{x^{*} \in X^{*}}\left\{f^{*}\left(-x^{*}\right)+\left(A^{*} g^{*}\right)\left(x^{*}\right)\right\} \\
& =\inf _{x^{*} \in X^{*}}\left\{f^{*}\left(-x^{*}\right)+\inf \left\{g^{*}\left(y^{*}\right): A^{*} y^{*}=x^{*}\right\}\right\} \\
& =\inf _{y^{*} \in Y_{A}^{*}}\left\{f^{*}\left(-A^{*} y^{*}\right)+g^{*}\left(y^{*}\right)\right\} \\
& =-\sup _{y^{*} \in Y_{A}^{*}}\left\{-f^{*}\left(-A^{*} y^{*}\right)-g^{*}\left(y^{*}\right)\right\} \\
& =-v\left(D_{A}\right) .
\end{aligned}
$$

Definition 4.1. $f^{*} \square A^{*} g^{*}$ is said to be $A^{*}$-exact at 0 if there exists $x^{*} \in X^{*}$ such that $\left(f^{*} \square A^{*} g^{*}\right)(0)=f^{*}\left(-x^{*}\right)+\left(A^{*} g^{*}\right)\left(x^{*}\right)$ and the infimum in the definition of $\left(A^{*} g^{*}\right)\left(x^{*}\right)$ is attained.

Definition 4.2. The triple $(f, g, A)$ is said to satisfy the condition $(F R C)_{A}$ if

$$
(f+g \circ A)^{*}(0) \geq\left(f^{*} \square A^{*} g^{*}\right)(0),
$$

and $f^{*} \square A^{*} g^{*}$ is $A^{*}$-exact at 0 .

Remark 4.1. (a) By (4.6) and (4.7), we have the following equivalences:

$$
v\left(D_{A}\right)>-\infty \Longleftrightarrow \operatorname{epi}\left(f^{*} \square A^{*} g^{*}\right) \cap(\{0\} \times \mathbb{R}) \neq \emptyset,
$$

Copyright (c) by SIAM. Unauthorized reproduction of this article is prohibited. 
and

$$
v\left(P_{A}\right)>-\infty \Longleftrightarrow \operatorname{epi}(f+g \circ A)^{*} \cap(\{0\} \times \mathbb{R}) \neq \emptyset .
$$

(b) The condition $(F R C)_{A}$ is different from the condition $\left(F R C_{A}\right)$ in [7, sect. 4], which was defined for lsc functions $f$ and $g$ and a continuous linear operator $A$, and states that $f^{*} \square A^{*} g^{*}$ is lsc and that the following equality holds:

$$
\text { epi }\left(f^{*} \square A^{*} g^{*}\right) \cap(\{0\} \times \mathbb{R})=\left(\text { epi } f^{*}+\left(A^{*} \times \operatorname{id}_{\mathbb{R}}\right)\left(\text { epi } g^{*}\right)\right) \cap(\{0\} \times \mathbb{R}) \text {. }
$$

We will see that when $f$ and $g$ are lsc and $A$ is continuous, our $(F R C)_{A}$ in Definition 4.2 is weaker than the condition above from [7, sect. 4].

(c) By (4.6), (4.7), and the weak dual inequality, (4.10) can be replaced by the equality $(f+g \circ A)^{*}(0)=\left(f^{*} \square A^{*} g^{*}\right)(0)$.

PROPOSITION 4.3. The following statements are equivalent:

(i) $(f, g, A)$ satisfies the condition $(F R C)_{A}$.

(ii) (4.10) and the following inclusion hold:

$$
\text { epi }\left(f^{*} \square A^{*} g^{*}\right) \cap(\{0\} \times \mathbb{R}) \subseteq\left(\text { epi } f^{*}+\left(A^{*} \times \operatorname{id}_{\mathbb{R}}\right)\left(\text { epi } g^{*}\right)\right) \cap(\{0\} \times \mathbb{R}) .
$$

(iii) The following inclusion holds:

$$
\text { epi }(f+g \circ A)^{*} \cap(\{0\} \times \mathbb{R}) \subseteq\left(\text { epi } f^{*}+\left(A^{*} \times \operatorname{id}_{\mathbb{R}}\right)\left(\text { epi } g^{*}\right)\right) \cap(\{0\} \times \mathbb{R}) .
$$

Proof. (i) $\Rightarrow$ (ii) Suppose that (i) holds. It suffices to show that (4.13) holds. To do this, take $(0, r) \in$ epi $\left(f^{*} \square A^{*} g^{*}\right) \cap(\{0\} \times \mathbb{R})$ (if the set on the left-hand side of (4.13) is empty, then the inclusion holds automatically). Then $\left(f^{*} \square A^{*} g^{*}\right)(0) \leq r$. By the condition $(F R C)_{A}, f^{*} \square A^{*} g^{*}$ is $A^{*}$-exact at 0 and, so, there exist $x^{*} \in X^{*}$ and $y^{*} \in Y_{A}^{*}$ such that $A^{*} y^{*}=x^{*},\left(A^{*} g^{*}\right)\left(x^{*}\right)=g^{*}\left(y^{*}\right)$, and

$$
f^{*}\left(-x^{*}\right)+\left(A^{*} g^{*}\right)\left(x^{*}\right)=f^{*}\left(-A^{*} y^{*}\right)+g^{*}\left(y^{*}\right) \leq r .
$$

Hence $g^{*}\left(y^{*}\right) \leq r-f^{*}\left(-A^{*} y^{*}\right)$ and $\left(A^{*} y^{*}, r-f^{*}\left(-A^{*} y^{*}\right)\right) \in\left(A^{*} \times \operatorname{id} \mathbb{R}_{\mathbb{R}}\right)\left(\right.$ epi $\left.g^{*}\right)$.

Moreover $\left(-A^{*} y^{*}, f^{*}\left(-A^{*} y^{*}\right)\right) \in$ epi $f^{*}$, and so,

$$
\begin{aligned}
(0, r) & =\left(-A^{*} y^{*}, f^{*}\left(-A^{*} y^{*}\right)\right)+\left(A^{*} y^{*}, r-f^{*}\left(-A^{*} y^{*}\right)\right) \\
& \in\left(\text { epi } f^{*}+\left(A^{*} \times \operatorname{id}_{\mathbb{R}}\right)\left(\text { epi } g^{*}\right)\right) \cap(\{0\} \times \mathbb{R}) .
\end{aligned}
$$

Thus (4.13) holds.

(ii) $\Rightarrow$ (iii) Suppose that (ii) holds. Then (4.10) and (4.13) hold. It is easy to see that (4.14) holds if the set on the left-hand side is empty. Let $(0, r) \in \operatorname{epi}(f+g \circ A)^{*} \cap$ $(\{0\} \times \mathbb{R})$, then $(f+g \circ A)^{*}(0) \leq r$. By $(4.10),\left(f^{*} \square A^{*} g^{*}\right)(0) \leq r$. This implies $(0, r) \in$ epi $\left(f^{*} \square A^{*} g^{*}\right) \cap(\{0\} \times \mathbb{R})$. By $(4.13),(0, r) \in\left(\right.$ epi $f^{*}+\left(A^{*} \times \operatorname{id} d_{\mathbb{R}}\right)\left(\right.$ epi $\left.\left.g^{*}\right)\right) \cap(\{0\} \times \mathbb{R})$, and (4.14) holds.

(iii) $\Rightarrow$ (i) Suppose that (4.14) holds. If $v\left(P_{A}\right)=-\infty$, then, by (4.6), (4.7), and the weak duality, $v\left(D_{A}\right)=-\infty$. Hence, the conclusion follows automatically. So, we assume that $v\left(P_{A}\right)=r \in \mathbb{R}$. By $(4.6),(f+g \circ A)^{*}(0)=-r$; hence $(0,-r) \in$ epi $(f+g \circ$ $A)^{*} \cap(\{0\} \times \mathbb{R})$. Applying (4.14), we have that $(0,-r) \in\left(\right.$ epi $f^{*}+\left(A^{*} \times \operatorname{id}_{\mathbb{R}}\right)\left(\right.$ epi $\left.\left.g^{*}\right)\right) \cap$ $(\{0\} \times \mathbb{R})$. Therefore, there exist $\left(x_{1}^{*}, r_{1}\right) \in$ epi $f^{*}$ and $\left(x_{2}^{*}, r_{2}\right) \in\left(A^{*} \times \mathrm{id}_{\mathbb{R}}\right)\left(\right.$ epi $\left.g^{*}\right)$ such that

$$
x_{1}^{*}+x_{2}^{*}=0 \quad \text { and } \quad r_{1}+r_{2}=-r .
$$

Copyright $@$ by SIAM. Unauthorized reproduction of this article is prohibited. 
Moreover, there exists $\bar{y}^{*} \in Y_{A}^{*}$ such that $A^{*} \bar{y}^{*}=x_{2}^{*}$ and $\left(\bar{y}^{*}, r_{2}\right) \in$ epi $g^{*}$. Consequently, $f^{*}\left(x_{1}^{*}\right) \leq r_{1}$ and $g^{*}\left(\bar{y}^{*}\right) \leq r_{2}$, and therefore,

$$
f^{*}\left(-x_{2}^{*}\right)+g^{*}\left(\bar{y}^{*}\right)=f^{*}\left(x_{1}^{*}\right)+g^{*}\left(\bar{y}^{*}\right) \leq r_{1}+r_{2}=-r=-v\left(P_{A}\right) .
$$

Noting that $\left(A^{*} g^{*}\right)\left(x_{2}^{*}\right) \leq g^{*}\left(\bar{y}^{*}\right)$ and using the weak dual inequality, we get from (4.16) that

$$
f^{*}\left(-x_{2}^{*}\right)+\left(A^{*} g^{*}\right)\left(x_{2}^{*}\right) \leq f^{*}\left(-x_{2}^{*}\right)+g^{*}\left(\bar{y}^{*}\right) \leq-v\left(P_{A}\right) \leq-v\left(D_{A}\right) .
$$

By (4.7) and the definition of the infimal convolution, one observes that

$$
-v\left(D_{A}\right)=\left(f^{*} \square A^{*} g^{*}\right)(0) \leq f^{*}\left(-x_{2}^{*}\right)+\left(A^{*} g^{*}\right)\left(x_{2}^{*}\right) .
$$

Combining this and (4.17), one gets

$$
\left(f^{*} \square A^{*} g^{*}\right)(0)=f^{*}\left(-x_{2}^{*}\right)+\left(A^{*} g^{*}\right)\left(x_{2}^{*}\right)=f^{*}\left(-x_{2}^{*}\right)+g^{*}\left(\bar{y}^{*}\right) .
$$

This means that $\left(f^{*} \square A^{*} g^{*}\right)(0)=-v\left(P_{A}\right)=(f+g \circ A)^{*}(0)$ by (4.6) and $f^{*} \square A^{*} g^{*}$ is $A^{*}$-exact at 0 . The proof is complete.

Remark 4.2. Note that the converse inclusions in (4.13) and (4.14) hold automatically. Hence, the inclusions (4.13) and (4.14) can be replaced by equalities.

The following theorem shows that the condition $(F R C)_{A}$ is equivalent to the strong Fenchel duality.

THEOREM 4.4. The triple $(f, g, A)$ satisfies the condition $(F R C)_{A}$ if and only if $v\left(P_{A}\right)=v\left(D_{A}\right)$ and $\left(D_{A}\right)$ has an optimal solution.

Proof. $(\Rightarrow)$ Suppose that $(f, g, A)$ satisfies the condition $(F R C)_{A}$. Then, by Remark 4.1(c),

$$
\left(f^{*} \square A^{*} g^{*}\right)(0)=(f+g \circ A)^{*}(0) .
$$

This together with (4.6) and (4.7) implies that $v\left(P_{A}\right)=v\left(D_{A}\right)$.

Now we shall prove that $\left(D_{A}\right)$ has an optimal solution. In fact, since $f^{*} \square A^{*} g^{*}$ is $A^{*}$-exact at 0 , there exist $\bar{x}^{*} \in X^{*}$ and $\bar{y}^{*} \in Y^{*}$ with $A^{*} \bar{y}^{*}=\bar{x}^{*}$ such that

$$
\begin{aligned}
-v\left(D_{A}\right) & =\left(f^{*} \square A^{*} g^{*}\right)(0)=f^{*}\left(-\bar{x}^{*}\right)+\left(A^{*} g^{*}\right)\left(\bar{x}^{*}\right) \\
& =f^{*}\left(-A^{*} \bar{y}^{*}\right)+g^{*}\left(\bar{y}^{*}\right) .
\end{aligned}
$$

This means that $\bar{y}^{*}$ is an optimal solution of $\left(D_{A}\right)$.

$(\Leftarrow)$ Suppose that $v\left(P_{A}\right)=v\left(D_{A}\right)$ and that $\left(D_{A}\right)$ has an optimal solution. By Proposition 4.3, it suffices to show that (4.14) holds. To this aim, let $(0, r) \in$ epi $(f+g \circ$ $A)^{*} \cap(\{0\} \times \mathbb{R})\left(\right.$ if epi $(f+g \circ A)^{*} \cap(\{0\} \times \mathbb{R})=\emptyset$, the result holds automatically). Then $-v\left(P_{A}\right)=(f+g \circ A)^{*}(0) \leq r$. Since $v\left(P_{A}\right)=v\left(D_{A}\right)$ and $\left(D_{A}\right)$ has an optimal solution, it follows that $-v\left(D_{A}\right) \leq r$ and that there exists $\bar{y}^{*} \in Y_{A}^{*}$ such that $-f^{*}\left(-A^{*} \bar{y}^{*}\right)-$ $g^{*}\left(\bar{y}^{*}\right)=v\left(D_{A}\right)$. Hence, one has that

$$
g^{*}\left(\bar{y}^{*}\right) \leq r-f^{*}\left(-A^{*} \bar{y}^{*}\right) .
$$

This means $\left(A^{*} \bar{y}^{*}, r-f^{*}\left(-A^{*} \bar{y}^{*}\right)\right) \in\left(A^{*} \times \operatorname{id}_{\mathbb{R}}\right)\left(\right.$ epi $\left.g^{*}\right)$.

Moreover, it is obvious that $\left(-A^{*} \bar{y}^{*}, f^{*}\left(-A^{*} \bar{y}^{*}\right)\right) \in$ epi $f^{*}$ and

$$
(0, r)=\left(-A^{*} \bar{y}^{*}, f^{*}\left(-A^{*} \bar{y}^{*}\right)\right)+\left(A^{*} \bar{y}^{*}, r-f^{*}\left(-A^{*} \bar{y}^{*}\right)\right) .
$$

Copyright (c) by SIAM. Unauthorized reproduction of this article is prohibited. 
One finds $(0, r) \in\left(\right.$ epi $f^{*}+\left(A^{*} \times \operatorname{id}_{\mathbb{R}}\right)\left(\right.$ epi $\left.\left.g^{*}\right)\right) \cap(\{0\} \times \mathbb{R})$, and (4.14) holds. The proof is complete.

For the remainder of this paper, $p \in X^{*}$ stands for the functional $p(\cdot):=\langle p, \cdot\rangle$, and its corresponding conjugate function is $p^{*}=\delta_{\{p\}}$.

THEOREM 4.5. $(f, g, A)$ satisfies the condition $(C C)_{A}$ if and only if for each $p \in X^{*},(f+p, g, A)$ satisfies the condition $(F R C)_{A}$.

Proof. By Proposition 4.3, to prove this result, it suffices to prove that $(C C)_{A}$ is equivalent to

$$
\text { epi }(f+p+g \circ A)^{*} \cap(\{0\} \times \mathbb{R})=\left(\operatorname{epi}(f+p)^{*}+\left(A^{*} \times \operatorname{id} \mathbb{R}\right)\left(\operatorname{epi} g^{*}\right)\right) \cap(\{0\} \times \mathbb{R})
$$

holds for each $p \in X^{*}$.

Let $p \in X^{*}$. It is easy to show that epi $(h+p)^{*}=\operatorname{epi} h^{*}+(p, 0)$ for any proper function $h$. Thus, one has that epi $(f+p+g \circ A)^{*}=\operatorname{epi}(f+g \circ A)^{*}+(p, 0)$, and so

$$
\text { epi }(f+p+g \circ A)^{*} \cap(\{0\} \times \mathbb{R})=\operatorname{epi}(f+g \circ A)^{*} \cap(\{-p\} \times \mathbb{R})+(p, 0) .
$$

Similarly, we have that

$$
\left(\text { epi }(f+p)^{*}+\left(A^{*} \times \operatorname{id}_{\mathbb{R}}\right)\left(\text { epi } g^{*}\right)\right) \cap(\{0\} \times \mathbb{R})=\left(\text { epi } f^{*}+\left(A^{*} \times \operatorname{id}_{\mathbb{R}}\right)\left(\text { epi } g^{*}\right)\right)
$$

Therefore, (4.18) holds if and only if

$$
\text { epi }(f+g \circ A)^{*} \cap(\{-p\} \times \mathbb{R})=\left(\text { epi } f^{*}+\left(A^{*} \times \operatorname{id}_{\mathbb{R}}\right)\left(\text { epi } g^{*}\right)\right) \cap(\{-p\} \times \mathbb{R}) .
$$

Consequently, (4.18) holds for each $p \in X^{*}$ if and only if (3.4) holds, i.e., $(C C)_{A}$ holds, and the proof is complete.

By Theorem 4.4 and Theorem 4.5, we get the following theorem straightforwardly:

TheOrem 4.6. The condition $(C C)_{A}$ holds if and only if for each $p \in X^{*}$, $v\left(P_{(A, p)}\right)=v\left(D_{(A, p)}\right)$, and $\left(D_{(A, p)}\right)$ has an optimal solution.

Corollary 4.7. Suppose that the condition $(C C)_{A}$ holds. Then $v\left(P_{A}\right)=v\left(D_{A}\right)$ and $\left(D_{A}\right)$ has an optimal solution.

Remark 4.3. Let $C$ be a convex subset of $X$. Recall from [2] that the quasirelative interior and the quasi-interior of $C$ are defined, respectively, by

qri $C:=\{x \in C: \operatorname{cl} \operatorname{cone}(C-x)$ is linear $\}$ and qi $C:=\{x \in C: \operatorname{cl} \operatorname{cone}(C-x)=X\}$.

Bot et al. established in [2, Theorem 3.14] the strong Fenchel duality between problems $\left(P_{A}\right)$ and $\left(D_{A}\right)$ under the following interiority condition:

$$
\begin{gathered}
0 \in \operatorname{qri}(A(\operatorname{dom} f)-\operatorname{dom} g), \\
0 \in \operatorname{qi}[(A(\operatorname{dom} f)-\operatorname{dom} g)-(A(\operatorname{dom} f)-\operatorname{dom} g)], \\
(0,0) \notin \text { qri co }\left[\left(\left(A \times \operatorname{id}_{R}\right)(\operatorname{epi} f)-\widehat{\operatorname{epi}}(g-v(P))\right) \cup\{(0,0)\}\right],
\end{gathered}
$$

where $\widehat{\text { epi }} g:=\{(x, r) \in X \times \mathbb{R}:(x,-r) \in$ epi $g\}$. It would be helpful to make some comparison among conditions $(F R C)_{A},(C C)_{A}$, and the interiority condition (4.21). Clearly, combining Theorem 4.4 and [2, Theorem 3.14], we see that the interiority condition (4.21) implies the condition $(F R C)_{A}$ but the converse is not true. Furthermore, in finite dimensional spaces, the interiority condition (4.21) implies $(C C)_{A}$ by [21, Theorem 2.8.3] (or [7, Theorem 3.1] and its proof), while the converse does not 
hold. For example, let $f, g: \mathbb{R} \rightarrow \overline{\mathbb{R}}$ be defined by $f=\delta_{\mathbb{R}_{+}}$and $g=\delta_{\mathbb{R}_{-}}$, respectively, and take $A=\mathrm{id}$. Then it is easy to see that $(C C)_{A}$ holds and so the condition $(F R C)_{A}$, but the above interiority condition does not hold as $0 \notin$ qri $(\operatorname{dom} f-\operatorname{dom} g)$. Hence, our Corollary 4.7 can be applied but not [2, Theorem 3.14]. Another such example in $\mathbb{R}^{2}$ is given in Example 4.1 below. However, in infinite dimensional spaces, to establish if condition (4.21) implies $(C C)_{A}$ is a hard problem and, so, we leave it as an open problem.

Example 4.1. Let $X=Y=\mathbb{R}^{2}$ and $A=\operatorname{id}_{X}$. Let

$$
\begin{aligned}
Z & =\left\{\left(t_{1}, t_{2}\right) \in \mathbb{R}^{2}: t_{2}-t_{1}<1, t_{1}+t_{2}>-1, t_{1} \geq 0\right\}, \\
W & =\left\{\left(t_{1}, t_{2}\right) \in \mathbb{R}^{2}: t_{1}+t_{2}<1, t_{1}-t_{2}<1, t_{1} \leq 0\right\} .
\end{aligned}
$$

Then $Z$ and $W$ are convex sets, but they are not closed and, as a consequence of that, the functions

$$
f=\delta_{Z} \quad \text { and } \quad g=\delta_{W}
$$

are proper convex functions but not lsc. If

$$
Z^{*}=\left\{\left(s_{1}, s_{2}\right) \in \mathbb{R}^{2}: s_{1}+s_{2} \leq 0, s_{1}-s_{2} \leq 0\right\}
$$

and

$$
W^{*}=\left\{\left(s_{1}, s_{2}\right) \in \mathbb{R}^{2}: s_{1}+s_{2} \geq 0, s_{1}-s_{2} \geq 0\right\},
$$

we obtain

$$
f^{*}\left(x^{*}\right)= \begin{cases}\left|s_{2}\right|, & x^{*}=\left(s_{1}, s_{2}\right) \in Z^{*}, \\ +\infty, & \text { otherwise }\end{cases}
$$

and

$$
g^{*}\left(x^{*}\right)= \begin{cases}\left|s_{2}\right|, & x^{*}=\left(s_{1}, s_{2}\right) \in W^{*}, \\ +\infty, & \text { otherwise }\end{cases}
$$

Clearly, $Z \cap W=\left\{\left(t_{1}, t_{2}\right) \in \mathbb{R}^{2}: t_{1}=0, t_{2} \in(-1,1)\right\}$ and $f+g=\delta_{Z \cap W}$. Hence

$$
(f+g)^{*}\left(x^{*}\right)=\left|s_{2}\right| \quad \text { for each } x=\left(s_{1}, s_{2}\right) \in \mathbb{R}^{2} .
$$

Consequently,

$$
\begin{aligned}
& \text { epi } f^{*}=\left\{\left(s_{1}, s_{2}, r\right):\left(s_{1}, s_{2}\right) \in Z^{*},\left|s_{2}\right| \leq r\right\}, \\
& \text { epi } g^{*}=\left\{\left(s_{1}, s_{2}, r\right):\left(s_{1}, s_{2}\right) \in W^{*},\left|s_{2}\right| \leq r\right\},
\end{aligned}
$$

and

$$
\text { epi }(f+g)^{*}=\left\{\left(s_{1}, s_{2}, r\right):\left|s_{2}\right| \leq r\right\} .
$$

Then, it is easy to check that epi $(f+g)^{*}=$ epi $f^{*}+$ epi $g^{*}$; hence the condition $(C C)_{A}$ holds. Thus Corollary 4.7 applies and the strong Fenchel duality holds. However, since

$$
\operatorname{cl}(\operatorname{cone}(\operatorname{dom} f-\operatorname{dom} g))=\left\{\left(t_{1}, t_{2}\right): t_{2} \geq 0\right\},
$$

it follows that $(0,0) \notin \operatorname{qri}(\operatorname{dom} f-\operatorname{dom} g)$. This means that the interiority condition (4.21) does not hold and so [2, Theorem 3.14] does not apply. 
5. Stable total Fenchel duality. Recall that $v\left(P_{(A, p)}\right)$ and $v\left(D_{(A, p)}\right)$ denote the optimal values of the problems $\left(P_{(A, p)}\right)$ and $\left(D_{(A, p)}\right)$, respectively, defined by (4.1) and (4.2). In this section we present a new necessary and sufficient condition for the stable total Fenchel duality, which states that $v\left(P_{(A, p)}\right)=v\left(D_{(A, p)}\right)$, and both problems $\left(P_{(A, p)}\right)$ and $\left(D_{(A, p)}\right)$ have optimal solutions, whichever $p \in X^{*}$ we take.

For each $p \in X^{*}$, let $S_{P}(p)$ denote the optimal set of $\left(P_{(A, p)}\right)$. Let $x_{0} \in(\operatorname{dom} f) \cap$ $A^{-1}(\operatorname{dom} g)$. Recall (cf. [17, p. 47]) that the Moreau-Rockafellar formula holds at $x_{0}$ if

$$
\partial(f+g \circ A)\left(x_{0}\right)=\partial f\left(x_{0}\right)+A^{*} \partial g\left(A x_{0}\right) .
$$

We say that the Moreau-Rockafellar formula is globally satisfied if it holds at each point $x_{0} \in(\operatorname{dom} f) \cap A^{-1}(\operatorname{dom} g)$.

Remark 5.1. The Moreau-Rockafellar formula is somewhat similar to the condition $G B C Q_{1}(f, \mathcal{A})$, which was introduced in [5] to study the Lagrange and FenchelLagrange dualities for conical programming. Clearly, in the case when $A=\operatorname{id}_{X}$, $g=\delta_{\mathcal{A}}$, and the condition $G B C Q_{1}(0, \mathcal{A})$ is satisfied, the Moreau-Rockafellar formula (5.1) is equivalent to the condition $G B C Q_{1}(f, \mathcal{A})$ in $[5]$ (see $[4,5]$ for details).

TheOREM 5.1. Let $x_{0} \in(\operatorname{dom} f) \cap A^{-1}(\operatorname{dom} g)$. Then the following conditions are equivalent:

(i) The Moreau-Rockafellar formula holds at $x_{0}$.

(ii) For each $p \in X^{*}$ such that $x_{0} \in S_{P}(p)$,

$$
\min _{x \in X}\{f(x)+g(A x)-\langle p, x\rangle\}=\max _{y^{*} \in Y_{A}^{*}}\left\{-f^{*}\left(p-A^{*} y^{*}\right)-g^{*}\left(y^{*}\right)\right\} .
$$

Proof. (i) $\Rightarrow$ (ii) Suppose that (i) holds. Let $p \in X^{*}$ be such that

$$
f\left(x_{0}\right)+g\left(A x_{0}\right)-\left\langle p, x_{0}\right\rangle=v\left(P_{(A, p)}\right) .
$$

Then, by [21, Theorem 2.5.7], $0 \in \partial(f-p+g \circ A)\left(x_{0}\right)$, which is equivalent to $p \in$ $\partial(f+g \circ A)\left(x_{0}\right)$. By (5.1), we have $p \in \partial f\left(x_{0}\right)+A^{*} \partial g\left(A x_{0}\right)$. Therefore, there exist $p_{1} \in \partial f\left(x_{0}\right)$ and $p_{2} \in \partial g\left(A x_{0}\right)$ such that

$$
p=p_{1}+A^{*} p_{2} .
$$

Applying (2.2), one has

$$
f^{*}\left(p_{1}\right)+f\left(x_{0}\right)=\left\langle p_{1}, x_{0}\right\rangle \text { and } g^{*}\left(p_{2}\right)+g\left(A x_{0}\right)=\left\langle p_{2}, A x_{0}\right\rangle .
$$

Noting that $\left\langle p_{2}, A x_{0}\right\rangle=\left\langle A^{*} p_{2}, x_{0}\right\rangle$ and using (5.3), it follows that

$$
f^{*}\left(p_{1}\right)+g^{*}\left(p_{2}\right)+f\left(x_{0}\right)+g\left(A x_{0}\right)=\left\langle p_{1}, x_{0}\right\rangle+\left\langle p_{2}, A x_{0}\right\rangle=\left\langle p, x_{0}\right\rangle .
$$

Therefore,

$$
-f^{*}\left(p_{1}\right)-g^{*}\left(p_{2}\right)=f\left(x_{0}\right)+g\left(A x_{0}\right)-\left\langle p, x_{0}\right\rangle=v\left(P_{(A, p)}\right) .
$$

By the weak dual inequality, $v\left(D_{(A, p)}\right) \leq v\left(P_{(A, p)}\right)$. On the other hand, since $p_{2} \in Y_{A}^{*}$,

$$
v\left(D_{(A, p)}\right) \geq-f^{*}\left(p-A^{*} p_{2}\right)-g^{*}\left(p_{2}\right)=-f^{*}\left(p_{1}\right)-g^{*}\left(p_{2}\right)=v\left(P_{(A, p)}\right),
$$

where the equalities hold because of (5.3) and (5.4). It follows that $v\left(D_{(A, p)}\right)=$ $v\left(P_{(A, p)}\right)$ and $p_{2}$ is a solution of the problem $\left(D_{(A, p)}\right)$. 
(ii) $\Rightarrow$ (i) Suppose that (ii) holds and take $p \in \partial(f+g \circ A)\left(x_{0}\right)$. Then $x_{0} \in S_{P}(p)$ and, because of (5.2),

$$
f\left(x_{0}\right)+g\left(A x_{0}\right)-\left\langle p, x_{0}\right\rangle=\max _{y^{*} \in Y_{A}^{*}}\left\{-f^{*}\left(p-A^{*} y^{*}\right)-g^{*}\left(y^{*}\right)\right\} .
$$

(5.2) also implies the existence of $q \in Y_{A}^{*}$ such that

$$
f\left(x_{0}\right)+g\left(A x_{0}\right)-\left\langle p, x_{0}\right\rangle=-f^{*}\left(p-A^{*} q\right)-g^{*}(q) .
$$

Rewriting the above equality and using the definition of the conjugate function (noting that $\left\langle q, A x_{0}\right\rangle=\left\langle A^{*} q, x_{0}\right\rangle$ ), we get

$$
0 \leq g^{*}(q)+g\left(A x_{0}\right)-\left\langle q, A x_{0}\right\rangle=\left\langle p-A^{*} q, x_{0}\right\rangle-f\left(x_{0}\right)-f^{*}\left(p-A^{*} q\right) \leq 0 .
$$

Hence,

$$
g^{*}(q)+g\left(A x_{0}\right)-\left\langle q, A x_{0}\right\rangle=0 \quad \text { and } \quad\left\langle p-A^{*} q, x_{0}\right\rangle-f\left(x_{0}\right)-f^{*}\left(p-A^{*} q\right)=0 .
$$

This implies that $q \in \partial g\left(x_{0}\right)$ and $p-A^{*} q \in \partial f\left(x_{0}\right)$. Consequently, $p=p-A^{*} q+A^{*} q \in$ $\partial f\left(x_{0}\right)+A^{*} \partial g\left(x_{0}\right)$, and the set on the left-hand side of (5.1) is contained in the set on the right-hand side. This completes the proof because the converse inclusion holds automatically.

The following theorem constitutes a global version of Theorem 5.1.

TheOREM 5.2. The Moreau-Rockafellar is globally satisfied if and only if (5.2) holds for each $p \in X^{*}$ such that $S_{P}(p) \neq \emptyset$.

Proof. $(\Rightarrow)$ It is a direct consequence of the implication (i) $\Rightarrow$ (ii) of Theorem 5.1.

$(\Leftarrow)$ Let $x_{0} \in(\operatorname{dom} f) \cap A^{-1}(\operatorname{dom} g)$. Then, $(5.2)$ holds by the assumption if there is $p \in X^{*}$ such that $x_{0} \in S_{P}(p)$, and the Moreau-Rockafellar formula is satisfied at $x_{0}$ by Theorem 5.1 .

Alternatively, if there is no $p \in X^{*}$ such that $x_{0} \in S_{P}(p)$, we get $\partial(f+g \circ A)\left(x_{0}\right)=$ $\emptyset$ and (5.1) is trivially satisfied. The proof is complete.

Taking $p=0$ in Theorem 5.1(ii), we get the following corollary.

Corollary 5.3. Suppose that $x_{0} \in S_{P}(0)$ and that the Moreau-Rockafellar formula holds at $x_{0}$. Then the total Fenchel duality holds:

$$
\min _{x \in X}\{f(x)+g(A x)\}=\max _{y^{*} \in Y_{A}^{*}}\left\{-f^{*}\left(-A^{*} y^{*}\right)-g^{*}\left(y^{*}\right)\right\} .
$$

Combining Corollary 5.3 and Proposition 3.11, we get the following corollary.

Corollary 5.4. Suppose that the condition $(C C)_{A}$ holds. If the primal problem $\left(P_{A}\right)$ has a solution, then the total Fenchel duality (5.5) holds.

6. Converse Fenchel duality. Recall that $f: X \rightarrow \overline{\mathbb{R}}, g: Y \rightarrow \overline{\mathbb{R}}$ are proper convex (not necessarily lsc) functions and that $A: X \rightarrow Y$ is a linear operator (not necessarily continuous) such that $A(\operatorname{dom} f) \cap \operatorname{dom} g \neq \emptyset$. Following [7], we further assume that

$$
0 \in \operatorname{dom}\left(f^{*}\right)+A^{*}\left(\operatorname{dom}\left(g^{*}\right)\right) .
$$

Consider the convex optimization problem $\left(P_{A}\right)$ and the corresponding dual problem $\left(D_{A}\right)$ as defined in (1.1) and (1.2), and recall that $v\left(P_{A}\right)$ and $v\left(D_{A}\right)$ denote their respective optimal values. The aim of this section is to study the existence of the 
so-called converse Fenchel duality for the triple $(f, g, A)$. This property is held when $v\left(P_{A}\right)=v\left(D_{A}\right)$ and $\left(P_{A}\right)$ has at least an optimal solution, that is,

$$
\min _{x \in X}\{f(x)+g(A x)\}=\sup _{y^{*} \in Y_{A}^{*}}\left\{-f^{*}\left(-A^{*} y^{*}\right)-g^{*}\left(y^{*}\right)\right\} .
$$

One possible approach is based on a fruitful idea applied by Bauschke in [1], Boţ and Wanka in [7], and $\mathrm{Ng}$ and Song in [16]. When $f$ and $g$ are proper, convex, lsc functions and $A$ is a continuous linear operator, the following equalities hold:

$$
\left(A^{*}\right)^{*}=A, \quad f^{* *}=f, \text { and } g^{* *}=g .
$$

If we define $\tilde{f}:=f^{*}, \tilde{g}:=g^{*}$, and $\tilde{A}:=A^{*}$, then the problem $\left(D_{A}\right)$ is equivalent to

$$
\left(\tilde{P}_{\tilde{A}}\right) \inf _{y^{*} \in Y^{*}}\left\{\tilde{f}\left(-\tilde{A} y^{*}\right)+\tilde{g}\left(y^{*}\right)\right\},
$$

and its associated dual is

$$
\left(\tilde{D}_{\tilde{A}}\right)-\sup _{x \in X^{* *}}\left\{-\tilde{g}^{*}\left(\tilde{A}^{*} x\right)-\tilde{f}^{*}(x)\right\}
$$

which is nothing else but the problem $\left(P_{A}\right)$, as is shown in [7]. Thus the converse Fenchel duality for problem $\left(P_{A}\right)$ is equivalent to the Fenchel duality for problem $\left(\tilde{P}_{\tilde{A}}\right)$ and so the results obtained in previous sections apply to derive conditions ensuring the existence of this converse duality. However, in the general case (i.e., when $f$ and $g$ are not assumed to be lsc and $A$ is not continuous) (6.2) does not hold; hence the results in previous sections cannot be applied directly, and the problem of the converse Fenchel duality becomes more interesting.

For each $p \in Y$, we define the following perturbed convex optimization problem

$$
\left(C P_{(A, p)}\right): \quad \inf _{x \in X}\{f(x)+g(p+A x)\},
$$

and the corresponding dual problem

$$
\left(C D_{(A, p)}\right): \quad \sup _{y^{*} \in Y_{A}^{*}}\left\{\left\langle p, y^{*}\right\rangle-f^{*}\left(-A^{*} y^{*}\right)-g^{*}\left(y^{*}\right)\right\} .
$$

$v\left(C P_{(A, p)}\right)$ and $v\left(C D_{(A, p)}\right)$ stand for the optimal values of problem $\left(C P_{(A, p)}\right)$ and $\left(C D_{(A, p)}\right)$, respectively, that is,

$$
v\left(C P_{(A, p)}\right)=\inf _{x \in X}\{f(x)+g(p+A x)\}
$$

and

$$
v\left(C D_{(A, p)}\right)=\sup _{y^{*} \in Y_{A}^{*}}\left\{\left\langle p, y^{*}\right\rangle-f^{*}\left(-A^{*} y^{*}\right)-g^{*}\left(y^{*}\right)\right\}
$$

It is easy to check that the weak dual inequality holds:

$$
v\left(C D_{(A, p)}\right) \leq v\left(C P_{(A, p)}\right) \quad \text { for each } p \in Y \text {. }
$$

Moreover, according to the definitions, we have the following expressions:

$$
v\left(C P_{(A, p)}\right)=(g \square(-A) f)(p)
$$


and

$$
v\left(C D_{(A, p)}\right)=\left(g^{*}+f^{*} \circ\left(-A^{*}\right)\right)_{A}^{*}(p),
$$

where we have defined

$$
\left(g^{*}+f^{*} \circ\left(-A^{*}\right)\right)_{A}^{*}(p):=\sup _{y^{*} \in Y_{A}^{*}}\left\{\left\langle p, y^{*}\right\rangle-f^{*}\left(-A^{*} y^{*}\right)-g^{*}\left(y^{*}\right)\right\} .
$$

In fact, (6.9) comes from the definitions, while (6.8) holds because

$$
\begin{aligned}
(g \square(-A) f)(p) & =\inf _{y \in Y}\{g(p-y)+(-A f)(y)\} \\
& =\inf _{y \in Y}\{g(p-y)+\inf \{f(x):-A x=y, x \in X\}\} \\
& =\inf _{x \in X}\{g(p+A x)+f(x)\} \\
& =v\left(C P_{(A, p)}\right) .
\end{aligned}
$$

In particular, when $p=0$, problems $\left(C P_{(A, p)}\right)$ and $\left(C D_{(A, p)}\right)$ are just the primal problem $\left(P_{A}\right)$ and its Fenchel dual $\left(D_{A}\right)$, and by (6.8) and (6.9):

$$
v\left(P_{A}\right)=(g \square(-A) f)(0)
$$

and

$$
v\left(D_{A}\right)=\left(g^{*}+f^{*} \circ\left(-A^{*}\right)\right)_{A}^{*}(0) .
$$

Parallel to Definitions 4.1 and 4.2 , we introduce the following converse notions.

Definition 6.1. $g \square(-A) f$ is said to be $A$-exact at 0 if there exists $y \in Y$ such that $(g \square(-A) f)(0)=g(-y)+(-A f)(y)$ and the infimum in the definition of $(-A f)(y)$ is attained.

Definition 6.2. The triple $(f, g, A)$ is said to satisfy the converse $(F R C)_{A}$ if

$$
\left(g^{*}+f^{*} \circ\left(-A^{*}\right)\right)_{A}^{*}(0) \geq(g \square(-A) f)(0)
$$

and $g \square(-A) f$ is $A$-exact at 0 .

Remark 6.1. (a) Converse $(F R C)_{A}$ is different from the condition $\left(C F R C_{A}\right)$ in [7, sect. 5], which was defined for lsc functions $f$ and $g$ and a continuous linear operator $A$, and states that $g \square(-A) f$ is lsc, and the following equality holds:

$$
\text { epi }(g \square(-A) f) \cap(\{0\} \times \mathbb{R})=\left(\text { epi } g+\left((-A) \times \operatorname{id}_{\mathbb{R}}\right)(\text { epi } f)\right) \cap(\{0\} \times \mathbb{R}) \text {. }
$$

It will be seen that the converse $(F R C)_{A}$ in Definition 6.2 is weaker than the one above (given in $[7$, sect. 5]).

(b) By $(6.10),(6.11)$, and the weak dual inequality, (6.12) can be replaced by the equality $\left(g^{*}+f^{*} \circ\left(-A^{*}\right)\right)_{A}^{*}(0)=(g \square(-A) f)(0)$.

(c) As shown in [7], the assumption $A(\operatorname{dom} f) \cap \operatorname{dom} g \neq \emptyset$ and (6.1) guarantee the following relations:

$$
-\infty<v\left(D_{A}\right) \leq v\left(P_{A}\right)<+\infty
$$

The proofs of Proposition 6.3 and Theorem 6.4 below are similar to the ones of Proposition 4.3 and Theorem 4.4, respectively, and we shall omit them here.

Proposition 6.3. The following statements are equivalent:

(i) $(f, g, A)$ satisfies the converse $(F R C)_{A}$.

Copyright (C) by SIAM. Unauthorized reproduction of this article is prohibited. 
(ii) (6.12) and the following inclusion hold:

$$
\text { epi }(g \square(-A) f) \cap(\{0\} \times \mathbb{R}) \subseteq\left(\text { epi } g+\left((-A) \times \operatorname{id}_{\mathbb{R}}\right)(\text { epi } f)\right) \cap(\{0\} \times \mathbb{R}) .
$$

(iii) The following inclusion holds:

$(6.15)$ epi $\left(g^{*}+f^{*} \circ\left(-A^{*}\right)\right)_{A}^{*} \cap(\{0\} \times \mathbb{R}) \subseteq\left(\right.$ epi $g+\left((-A) \times \operatorname{id}_{\mathbb{R}}\right)($ epi $\left.f)\right) \cap(\{0\} \times \mathbb{R})$.

Remark 6.2. Note that the converse inclusions in (6.14) and (6.15) hold automatically. Hence, the inclusions (6.14) and (6.15) can be replaced by equalities.

TheOrem 6.4. The triple $(f, g, A)$ satisfies the converse $(F R C)_{A}$ if and only if $v\left(P_{A}\right)=v\left(D_{A}\right)$ and $\left(P_{A}\right)$ has an optimal solution.

Next we introduce the notion of converse $(C C)_{A}$.

Definition 6.5. The triple $(f, g, A)$ satisfies the converse $(C C)_{A}$ if

$$
\text { epi }\left(g^{*}+f^{*} \circ\left(-A^{*}\right)\right)_{A}^{*} \subseteq \text { epi } g+\left((-A) \times \operatorname{id}_{\mathbb{R}}\right)(\text { epi } f) .
$$

Remark 6.3. Note that the converse inclusion in (6.16) holds automatically. Hence, the inclusion (6.16) can be replaced by an equality.

TheOREm 6.6. $(f, g, A)$ satisfies the converse $(C C)_{A}$ if and only if for each $p \in Y$, we have $v\left(C P_{(A, p)}\right)=v\left(C D_{(A, p)}\right)$ and $\left(C P_{(A, p)}\right)$ has at least an optimal solution.

Proof. $(\Rightarrow)$ Let $p \in Y$ and $r=v\left(C D_{(A, p)}\right)$. Since $v\left(D_{A}\right) \in \mathbb{R}$ by Remark 6.1(c), it follows from (6.6) that (noting that $v\left(D_{A}\right)=v\left(C D_{(A, 0)}\right)$ )

$$
r \geq \sup _{y^{*} \in Y_{A}^{*}}\left\langle p, y^{*}\right\rangle-\sup _{y^{*} \in Y_{A}^{*}}\left(f^{*}\left(-A^{*} y^{*}\right)-g^{*}\left(y^{*}\right)\right)=\sup _{y^{*} \in Y_{A}^{*}}\left\langle p, y^{*}\right\rangle-v\left(D_{A}\right)>-\infty .
$$

Without loss of generality, we may assume that $r<+\infty$ (because the case when $r=+\infty$ is trivial). Thus $(p, r) \in$ epi $\left(g^{*}+f^{*} \circ\left(-A^{*}\right)\right)_{A}^{*}$ by (6.9). This together with (6.16) implies that $(p, r) \in$ epi $g+\left((-A) \times \operatorname{id}_{\mathbb{R}}\right)($ epi $f)$. Therefore, there exist $\left(y_{1}, r_{1}\right) \in$ epi $g$ and $\left(y_{2}, r_{2}\right) \in\left((-A) \times \operatorname{id}_{\mathbb{R}}\right)($ epi $f)$ such that

$$
y_{1}+y_{2}=p \quad \text { and } \quad r_{1}+r_{2}=r .
$$

Moreover, there exists $\bar{x} \in X$ such that $-A \bar{x}=y_{2}$ and $\left(\bar{x}, r_{2}\right) \in$ epi $f$. Consequently, $f(\bar{x}) \leq r_{2}$ and $g\left(y_{1}\right) \leq r_{1}$. Therefore,

$$
g(p+A \bar{x})+f(\bar{x})=g\left(p-y_{2}\right)+f(\bar{x})=g\left(y_{1}\right)+f(\bar{x}) \leq r_{1}+r_{2}=r .
$$

By the weak duality, $v\left(C P_{(A, p)}\right)=v\left(C D_{(A, p)}\right)$ and $\bar{x}$ is an optimal solution of $\left(C P_{(A, p)}\right)$.

$(\Leftarrow)$ Consider $p \in Y$ and $r \in \mathbb{R}$ such that $(p, r) \in$ epi $\left(g^{*}+f^{*} \circ\left(-A^{*}\right)\right)_{A}^{*}$. By (6.9), $v\left(C D_{(A, p)}\right)=\left(g^{*}+f^{*} \circ\left(-A^{*}\right)\right)_{A}^{*}(p) \leq r$.

Since $v\left(C P_{(A, p)}\right)=v\left(C D_{(A, p)}\right)$ and $\left(C P_{(A, p)}\right)$ has an optimal solution, it follows that $v\left(C P_{(A, p)}\right) \leq r$ as well as the existence of $\bar{x} \in X$ such that $f(\bar{x})+g(p+A \bar{x}) \leq r$. Hence, one gets

$$
f(\bar{x}) \leq r-g(p+A \bar{x}),
$$

which entails $(-A \bar{x}, r-g(p+A \bar{x})) \in\left((-A) \times \operatorname{id}_{\mathbb{R}}\right)($ epi $f)$.

Obviously, $(p+A \bar{x}, g(p+A \bar{x})) \in$ epi $g$, and

$$
(p, r)=(p+A \bar{x}, g(p+A \bar{x}))+(-A \bar{x}, r-g(p+A \bar{x})) .
$$

Copyright $@$ ㅇ by SIAM. Unauthorized reproduction of this article is prohibited. 
One sees that $(p, r) \in$ epi $g+\left((-A) \times \operatorname{id}_{\mathbb{R}}\right)($ epi $f)$. Hence (6.16) holds and the proof is complete.

The following corollary is straightforward.

Corollary 6.7. Suppose that the converse $(C C)_{A}$ holds. Then $v\left(P_{A}\right)=v\left(D_{A}\right)$ and $\left(P_{A}\right)$ has an optimal solution.

We now present a new necessary and sufficient condition for the stable total converse Fenchel duality, which states that

$$
\min _{x \in X}\{f(x)+g(p+A x)\}=\max _{y^{*} \in Y_{A}^{*}}\left\{\left\langle p, y^{*}\right\rangle-f^{*}\left(-A^{*} y^{*}\right)-g^{*}\left(y^{*}\right)\right\} \quad \text { for each } p \in Y,
$$

or equivalently, $v\left(C P_{(A, p)}\right)=v\left(C D_{(A, p)}\right)$ and both problems $\left(C P_{(A, p)}\right)$ and $\left(C D_{(A, p)}\right)$ have optimal solutions, whichever $p \in Y$ we take. For each $p \in Y$, let $S_{C D}(p)$ denote the solution set of the problem $\left(C D_{(A, p)}\right)$.

Definition 6.8. Let $y_{0}^{*} \in Y_{A}^{*} \cap\left(\operatorname{dom} g^{*}\right) \cap(-A)^{*}\left(\operatorname{dom} f^{*}\right)$. We say that the converse Moreau-Rockafellar formula holds at $y_{0}^{*}$ if

$$
\partial\left(f^{*} \circ\left(-A^{*}\right)+g^{*}\right)\left(y_{0}^{*}\right)=(-A) \partial f^{*}\left(-A^{*} y_{0}^{*}\right)+\partial g^{*}\left(y_{0}^{*}\right) .
$$

We say that the converse Moreau-Rockafellar formula is globally satisfied if it holds at each point $y_{0}^{*} \in Y_{A}^{*} \cap\left(\operatorname{dom} g^{*}\right) \cap(-A)^{*}\left(\operatorname{dom} f^{*}\right)$.

Similar to the proof of Theorem 5.1, we obtain the following theorem directly.

Theorem 6.9. Let $y_{0}^{*} \in Y_{A}^{*} \cap\left(\operatorname{dom} g^{*}\right) \cap(-A)^{*}\left(\operatorname{dom} f^{*}\right)$. Then the following conditions are equivalent:

(i) The converse Moreau-Rockafellar formula holds at $y_{0}^{*}$.

(ii) For each $p \in Y$ such that $y_{0}^{*} \in S_{C D}(p)$,

$$
\min _{x \in X}\{f(x)+g(p+A x)\}=\max _{y^{*} \in Y_{A}^{*}}\left\{\left\langle p, y^{*}\right\rangle-f^{*}\left(-A^{*} y^{*}\right)-g^{*}\left(y^{*}\right)\right\} .
$$

The following theorem is a global version of Theorem 6.9.

THEOREM 6.10. The converse Moreau-Rockafellar formula is globally satisfied if and only if (6.18) holds for each $p \in Y$ such that $S_{C D}(p) \neq \emptyset$.

Taking $p=0$ in Theorem 6.9 (ii), we get the following corollary.

Corollary 6.11. Suppose that $y_{0}^{*} \in S_{C D}(0)$ and that the converse MoreauRockafellar formula holds at $y_{0}^{*}$. Then, the total converse Fenchel duality holds:

$$
\min _{x \in X}\{f(x)+g(A x)\}=\max _{y^{*} \in Y_{A}^{*}}\left\{-f^{*}\left(A^{*} y^{*}\right)-g^{*}\left(y^{*}\right)\right\}=-f^{*}\left(A^{*} y_{0}^{*}\right)-g^{*}\left(y_{0}^{*}\right) .
$$

Proposition 6.12. Suppose that $(f, g, A)$ satisfies the converse $(C C)_{A}$. Then, for each $y^{*} \in Y_{A}^{*} \cap\left(\operatorname{dom} g^{*}\right) \cap(-A)^{*}\left(\operatorname{dom} f^{*}\right)$, the converse Moreau-Rockafellar formula holds at $y^{*}$.

Proof. Suppose that $(f, g, A)$ satisfies the converse $(C C)_{A}$, i.e., (6.16) holds. Let $y^{*} \in Y_{A}^{*} \cap\left(\operatorname{dom} g^{*}\right) \cap(-A)^{*}\left(\operatorname{dom} f^{*}\right)$ and $p \in \partial\left(f^{*} \circ\left(-A^{*}\right)+g^{*}\right)\left(y^{*}\right)$. Then we have

$$
\left(f^{*} \circ\left(-A^{*}\right)+g^{*}\right)_{A}^{*}(p)+\left(f^{*} \circ\left(-A^{*}\right)+g^{*}\right)\left(y^{*}\right)=\left\langle p, y^{*}\right\rangle .
$$

This implies that

$$
\left(p,\left\langle p, y^{*}\right\rangle-\left(f^{*} \circ\left(-A^{*}\right)+g^{*}\right)\left(y^{*}\right)\right) \in \operatorname{epi}\left(f^{*} \circ\left(-A^{*}\right)+g^{*}\right)_{A}^{*} .
$$

By (6.16),

$$
\left(p,\left\langle p, y^{*}\right\rangle-\left(f^{*} \circ\left(-A^{*}\right)+g^{*}\right)\left(y^{*}\right)\right) \in \operatorname{epi} g+\left((-A) \times \operatorname{id}_{\mathbb{R}}\right)(\text { epi } f) .
$$

Copyright $@$ ㅇ by SIAM. Unauthorized reproduction of this article is prohibited. 
Therefore, there exist $\left(y_{1}, r_{1}\right) \in$ epi $g$ and $\left(y_{2}, r_{2}\right) \in((-A) \times \operatorname{id} \mathbb{R})($ epi $f)$ such that

$$
y_{1}+y_{2}=p \text { and } r_{1}+r_{2}=\left\langle p, y^{*}\right\rangle-\left(f^{*} \circ\left(-A^{*}\right)+g^{*}\right)\left(y^{*}\right) .
$$

Moreover, there exists $\bar{x} \in X$ such that $-A \bar{x}=y_{2}$ and $\left(\bar{x}, r_{2}\right) \in$ epi $f$. Consequently, $f(\bar{x}) \leq r_{2}$ and $g\left(y_{1}\right) \leq r_{1}$. Therefore, by (6.20),

$$
f(\bar{x})+g\left(y_{1}\right) \leq r_{1}+r_{2}=\left\langle y_{1}+y_{2}, y^{*}\right\rangle-f^{*}\left(-A^{*} y^{*}\right)-g^{*}\left(y^{*}\right) .
$$

Rewriting the above equality and using the definition of the conjugate function (noting that $\left.\left\langle\bar{x},-A^{*} y^{*}\right\rangle=\left\langle-A \bar{x}, y^{*}\right\rangle=\left\langle y_{2}, y^{*}\right\rangle\right)$, we get

$$
0 \leq f(\bar{x})+f^{*}\left(-A^{*} y^{*}\right)-\left\langle\bar{x},-A^{*} y^{*}\right\rangle \leq\left\langle y_{1}, y^{*}\right\rangle-g^{*}\left(y^{*}\right)-g\left(y_{1}\right) \leq 0 .
$$

Hence,

$$
f(\bar{x})+f^{*}\left(-A^{*} y^{*}\right)-\left\langle\bar{x},-A^{*} y^{*}\right\rangle=0 \quad \text { and } \quad\left\langle y_{1}, y^{*}\right\rangle-g^{*}\left(y^{*}\right)-g\left(y_{1}\right)=0 .
$$

This implies that $\bar{x} \in \partial f^{*}\left(-A^{*} y^{*}\right)$ and $y_{1} \in \partial g^{*}\left(y^{*}\right)$. Consequently, $p=y_{1}+y_{2}=$ $-A \bar{x}+y_{1} \in(-A) \partial f^{*}\left(-A^{*} y^{*}\right)+\partial g^{*}\left(y^{*}\right)$, and the set on the left-hand side of (6.17) is contained in the set on the right-hand side. This completes the proof because the converse inclusion holds automatically.

Combining Corollary 6.11 and Proposition 6.12, we have the following corollary.

Corollary 6.13. Suppose that converse $(C C)_{A}$ holds. If the dual problem $\left(D_{A}\right)$ has an optimal solution, then the total converse Fenchel duality (6.19) holds.

Remark 6.4. All the considerations made in section 4 and section 6 remain valid even without assuming that $f$ and $g$ are convex.

Acknowledgment. The authors are grateful to both reviewers for their many helpful suggestions and remarks which improved the quality of the paper.

\section{REFERENCES}

[1] H. H. Bauschke, Proof of a conjecture by Deutsch, Li, and Swetits on duality of optimization problems, J. Optim. Theory Appl., 102 (1999), pp. 697-703.

[2] R. I. Boţ, E. R. Csetnek, and G. Wanka, Regularity conditions via quasi-relative interior in convex programming, SIAM. J. Optim., 19 (2008), pp. 217-233.

[3] R. I. Boţ, S. M. GRAD, And G. Wanka, Weaker Constraint Qualifications in Maximal Monotonicity, Numer. Funct. Anal. Optim., 28 (2007), pp. 27-41.

[4] R. I. Boț, S. M. GRAD, AND G. WANKA, On strong and total Lagrange duality for convex optimization problems, J. Math. Anal. Appl., 337 (2008), pp. 1315-1325.

[5] R. I. Boty, S. M. Grad, And G. Wanka, New regularity conditions for strong and total Fenchel-Lagrange duality in infinite dimensional spaces, Nonlinear Anal., 69 (2008), pp. 323-336.

[6] R. I. Boţ, I. B. Hodrea, And G. Wanka, $\epsilon$-optimality conditions for composed convex optimization problems, J. Approx. Theory, 153 (2008), pp. 108-121.

[7] R. I. Boţ AND G. WANKA, A weaker regularity condition for subdifferential calculus and Fenchel duality in infinite dimensional spaces, Nonlinear Anal., 64 (2006), pp. 2787-2804.

[8] J. M. Borwein, V. Jeyakumar, A. Lewis, And Wolkowicz, Constrained approximation via convex programming, preprint, University of Waterloo, Waterloo, Ontario, 1988.

[9] R. S. BurachiK and V. JEyAKUmar, A dual condition for the convex subdifferential sum formula with applications, J. Convex Anal., 12 (2005), pp. 279-290.

[10] R. S. Burachik and V. Jeyakumar, A simpler closure condition for the normal cone intersection formula, Proc. Amer. Math. Soc., 133 (2005), pp. 1741-1748.

[11] R. S. Burachik, V. Jeyakumar, and Z. Y. Wu, Necessary and sufficient conditions for stable conjugate duality, Nonlinear Anal., 64 (2006), pp. 1998-2006.

Copyright (c) by SIAM. Unauthorized reproduction of this article is prohibited. 
[12] B. D. Craven, Mathematical Programming and Control Theory, Chapman and Hall, London, 1978.

[13] H. Hantoute, M. A. López, And C. ZăLinescu, Subdifferential calculus rules in convex analysis: A unifying approach via pointwise supremum functions, SIAM J. Optim., 19 (2008), pp. 863-882.

[14] J. Hiriart-Urruty and C. Lemaréchal, Convex Analysis and Minimization Algorithms I, Vol. 305 of Grundlehren der Mathematschen Wissenschaften, Springer-Verlag, New York, 1993.

[15] R. B. Holmes, Geometric Functional Analysis, Springer-Verlag, Berlin, 1975.

[16] K. F. NG And W. Song, Fenchel duality in infinite-dimensional setting and its applications, Nonlinear Anal., 25 (2003), pp. 845-858.

[17] R. R. Phelps, Convex functions, monotone operators and differentiability, 2nd ed., Lecture Notes in Math. 1364, Springer-Verlag, Berlin, 1993.

[18] R. T. Rockafellar, An extension of Fenchel's duality theorem for convex functions, Duke Math. J., 33 (1966), pp. 81-90.

[19] R. T. Rockafellar, Conjugate duality and optimization, Conference Board of the Mathematical Sciences Regional Conference Series in Applied Mathematics 16, SIAM, Philadelphia, 1974.

[20] T. Strömberg, The operation of infimal convolution, Disscuss. Math., 352 (1996), pp. 1-61.

[21] C. ZăLInescu, Convex Analysis in General Vector Spaces, World Scientific, River Edge, New Jersey, 2002.

Copyright (c) by SIAM. Unauthorized reproduction of this article is prohibited. 